\title{
MODELING SYSTEM VARIATION
}

\author{
Ken Krechmer \\ International Center for Standards Research, 757 Greer Road, Palo Alto, CA 94303 USA \\ Email: krechmer@csrstds.com
}

Key words: System of constraints, order, Shannon entropy, communications systems, standards

\begin{abstract}
This paper proposes that the mathematical relationship between an entropy distribution and its limit offers some new insight into system performance. This relationship can be used to quantify variation among the entities of a system, caused by tolerance, options, specification or implementation errors, independent of noise, impact communications system performance. Means to address these variations are offered.
\end{abstract}

\section{INTRODUCTION}

C. Shannon in his seminal work, The Mathematical Theory of Communications (1963), describes a communications system as a "...system of constraints...". This paper proposes that Shannon's concept of a system of constraints describes a specific form of order that may be quantified. Order is defined as a fixed relationship; a special case in probability theory, where $p(\mathrm{a} \mid \mathrm{b})=p(\mathrm{~b} \mid \mathrm{a})=1$. The ordered nature of the system of constraints used for communications is clear when the relationship between the transmitter, transmission link and receiver is considered (see Figure 1). A properly designed communications system in the absence of noise, receives what is transmitted with a probability of one. Understanding the mathematical form of the system of constraints allows the quantification of what is a properly designed communications system.

\section{A MODEL OF A COMMUNI- CATIONS SYSTEM}

For communications to occur, any transmitter and receiver must be related by a specific order, whether it be human language (dictionaries and formal syntax maintain the order), ASCII characters, specific frequencies, voltages, or common protocols between a transmitter and receiver. In the last four examples order is maintained by reference to published documents which may be termed standards.
The philosopher I. Kant (1800) first elucidated the idea that a comparison is necessary for any form of understanding. As example, in the course of reading, a word appears of unknown meaning. The reader refers to a dictionary which defines the relationship between words and their meanings. Assuming that the author also uses a similar dictionary, the reader looks up the unknown word Upon finding the same word (a comparison), the reader now understands the meaning of the word. This three phase process, apply common reference, compare received signal to reference and identify signal, occurs in any communications process. In any communications system, order (in the form of a common reference) must exist between the transmitter and receiver to create the basis of comparison necessary before communications can occur.

Figure 1 diagrammatically shows the relationship between a system of constraints and a communications system. Each parameter of the transmitter and receiver that directly relates to each other and/or to the transmission link is a single set of constraints. The multiple parameters of the transmitter and receiver that directly relate to the transmission link and/or each other are the system of constraints (shown within the dotted line). Note that the system of constraints is not congruent with the Transmitter, Receiver or Transmission link in this communications system model. 


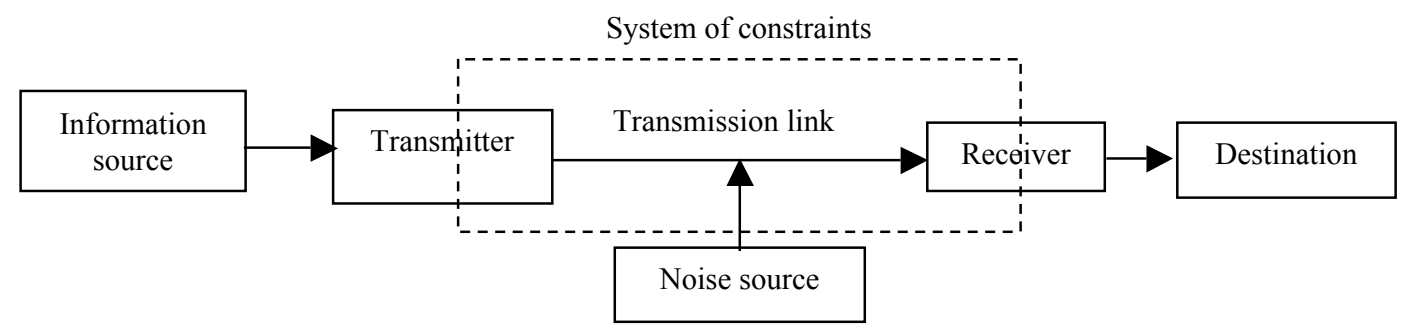

Figure 1: Communications system.

\section{A MODEL OF A SIMPLE INFORMATION CHANNEL}

Figure 2 (from Abramson [1963] plus the dashed rectangle S) presents a logical model of a simple information channel consisting of a transmitter of alphabet A with individual elements $a_{i}$ and total elements $\mathrm{t}$ and a receiver of alphabet $\mathrm{B}$ with individual elements $b_{i}$ and total elements $r$. Alphabet A and alphabet B are related by the existence of a common set of elements $\mathrm{S}$, where $\mathrm{S}=$ A $\square$ B. Without noise, when $S=A \square B$ and both $r$ and $\mathrm{t} \geq \mathrm{n}>0$ then $\mathrm{S}>0 . \mathrm{S}=\mathrm{A} \square \mathrm{B}>0$ are the set theoretic constraints that define the preexisting order in Figure 2.

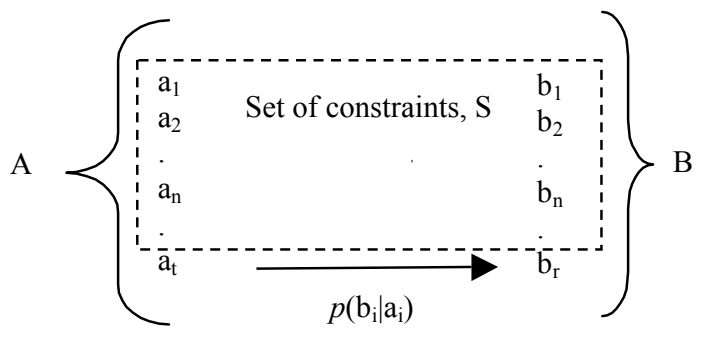

Figure 2: An information channel.

This model represents the transmission link as the probabilistic relationship between ordered pairs of elements. The transmission link's constraints only exist in the choice of the alphabets $\mathrm{A}$ and $\mathrm{B}$. The element pairs $a_{1}$ and $b_{1}, a_{2}$ and $b_{2}, \ldots a_{n}$ and $b_{n}$ are each defined as a preexisting ordered pair. B. Russell (no date) refers to this as a one-one relationship. When a transmission link connects one or more ordered pairs communications is possible. Without noise, the sets $\mathrm{A}$ and $\mathrm{B}$ are related by the ordered relationship of their elements where $p\left(\mathrm{~b}_{\mathrm{i}} \mid \mathrm{a}_{\mathrm{i}}\right)$ equals one, for ordered pairs 1 through $n$. The set of constraints termed $\mathrm{S}$ is formed by the order between the elements of sets $\mathrm{A}$ and $\mathrm{B}$ and the common alphabet size $n$. In Figure 2, the elements $a_{n+1}$ through $a_{t}$ and $b_{n+1}$ through $b_{r}$ are not ordered and therefore not included in the set of constraints.
An example of such an information channel is a human transmitter using the 26 letter English alphabet $a_{t}$ through $z_{t}$ and a human receiver using the same alphabet $a_{r}$ through $z_{r}$. One condition for error free communications is that the humans use a common alphabet. This condition is a set of constraints consisting of the 26 ordered pairs $a_{t}$ and $a_{r}, b_{t}$ and $b_{r}, \ldots . z_{t}$ and $z_{r}$. Using this set of constraints the humans are better able to communicate. Without one or more preexisting (before communications) ordered pairs, no reference exists and no comparisons are possible. When the same 26 letter alphabets are used by each person, communications based on the alphabet can occur.

The ordered pairs of a communications system may be created by chemical bonds (A-C, G-T in DNA), preexisting written or spoken alphabets, preexisting word dictionaries or the specifications of the transmitter and receiver (electronic communications) that constrain the implementation of the transmitter, transmission link, and receiver (entities). The definition of an entity here is arbitrary, and indicates some independence from other entities. The simplest entity is a single set (e.g., set A or set B); a complex entity may consist of multiple sets. The preexisting sets of constraints are the system of constraints defining the relationship between two or more entities. In a functioning communications system the implementations of the transmitter, transmission link and receiver are each bound by this system of constraints.

Note that the set of constraints shown in Figure 2 is geometrically congruent with the system of constraints shown in Figure 1. This suggests that Figure 2 offers the basis for a more rigorous description of the system of constraints.

\section{A MODEL OF THE BOUNDS ON A SET}

Describing the relationship between the transmitter and the receiver that enables communications first 
requires a description of each of these entities. In information theoretic terms the system of constraints defines the bounds of the information channel which includes the transmitter and receiver. Figure 2 models the simplest possible information channel between two entities as a single set of constraints, $\mathrm{S}$. Considering each entity as a single set, the information theoretic description of a single set (A) is:

$$
H(A)=\square \bigsqcup_{i=1}^{i=n} p\left(a_{i}\right) \log p\left(a_{i}\right)
$$

This equation describes the entropy distribution $(\mathrm{H})$ of set $\mathrm{A}$ with $\mathrm{n}$ discrete random variables $\mathrm{a}_{\mathrm{i}}$. The limit of $H(A)=\log n$ which is the bound of the entropy of set $A$ and $H(A)$ approaches this bound as a limit. The logarithm of the number of elements of the set along with the description of the set (in this case, set A), describes the set bounds in information theoretic terms.

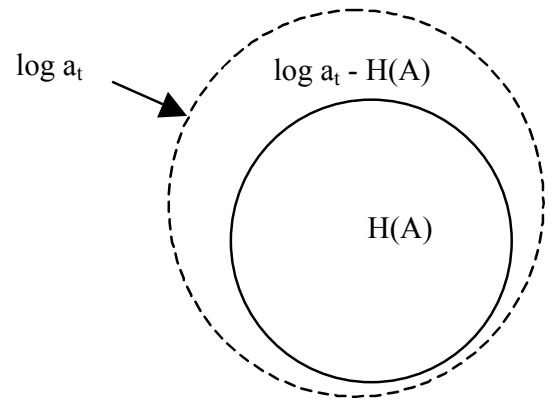

Figure 3: Venn diagram of an entropy distribution and its bound.

The relationship of an entropy distribution (e.g., from a transmitter or to a receiver) to its bound is shown in Figure 3. This relationship can be explored using the concept of mutual information. From Thomas and Cover (page 20) mutual information (MI) is defined as:

$$
M I=I(A ; B)=H(B)-H(B \mid A)
$$

Considering the entropy distribution $[\mathrm{H}(\mathrm{A})]$ in Figure 3 and its bound $\left(\log a_{t}\right)$ :

$$
\begin{aligned}
& H(B \mid A)=\log a_{t}-(H(A) \\
& M I=\log a_{t}-\left(\log a_{t}-H(A)\right)
\end{aligned}
$$

Therefore the mutual information, $I\left(A ; \log a_{t}\right)=$ $H(A)$. This explains that an entropy distribution when considered in its own context is the mutual information. A similar result is noted in Thomas and Cover (page 20) as self-information. However "its own context" may be either the bound of $\mathrm{H}(\mathrm{A})$, which must equal the maximum entropy distribution of $\mathrm{H}(\mathrm{A})$ or $\mathrm{H}(\mathrm{A})$ itself. If the context is not the bound of $\mathrm{H}(\mathrm{A})$ or $\mathrm{H}(\mathrm{A})$ itself, then there are two or more sets. The author proposes that a single entropy distribution must be considered in the context of its bound (e.g., $\log a_{t}$ ), as using the context $\mathrm{H}(\mathrm{A})$ is self-referential. This form of selfinformation is termed relative-information. This is an important point, the relative-information in a set only exists in context and the only logically consistent context is the limit of the entropy distribution. While this change in the context used to define relative-information does not change the value of mutual information, it has other ramifications that are developed below.

Figure 3 is also useful in defining the term similarity. By definition all sets that are similar to $\mathrm{H}(\mathrm{A})$ fall within the limit of $\mathrm{H}(\mathrm{A})$. For the purpose of creating a preexisting order (i.e., similarity), a specific description of a single entity (which may consist of multiple sets) may be made. This paper terms such a description a similarity description as the purpose of making such a description of an entity is almost always to create or maintain similar entities.

\section{A MODEL OF AN INFOR- MATION CHANNEL WITH BOUNDS}

Figure 1 defines a communications system. Focusing in, Figure 2 describes how an information channel exists within a communications system when the elements of two sets exist as ordered pairs across a transmission link. Figure 3 develops the relationship of a single entropy distribution to its bound. Figure 4 combines the concepts shown in Figure 2 with the relationships shown in Figure 3 to model an information channel and its bounds using Venn diagrams.

Figure 4 shows the case where the transmitter $(\mathrm{t})$ and receiver $(r)$ sets each have $n$ ordered pairs. Log $a_{t}$ is the bound of the transmitted entropy $[\mathrm{H}(\mathrm{A})]$ and $\log b_{r}$ is the bound of the received entropy [H(B)]. These bounds are shown as dotted concentric circles around the related entropy $\mathrm{H}(\mathrm{A})$ and $\mathrm{H}(\mathrm{B})$. Figure 4 is provided for visualization, not calculation, as the shapes are idealized. The entropy [H(A) and $\mathrm{H}(\mathrm{B})]$ always remains within its respective bound $\left(\log a_{t}\right.$ and $\log b_{r}$ ). 
Figure 4 models how the relationship between, and bounds on, $\mathrm{H}(\mathrm{A})$ and $\mathrm{H}(\mathrm{B})$ limit the maximum mutual information. MI (the area within the solid line lens in Figure 4) is the mutual information transferred across the information channel between the transmitter and the receiver.

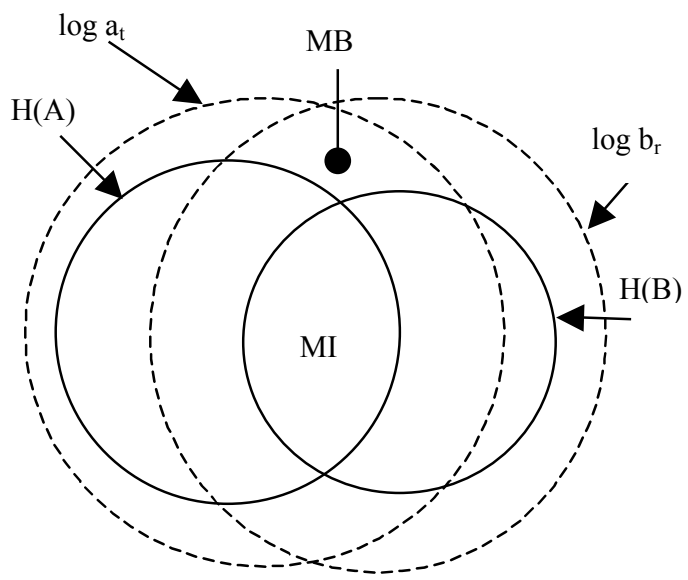

Figure 4: Venn diagram of an information channel and its bounds.

The mutual information (MI) transmission equation for the information channel shown in Figure 4 is:

$$
M I=I(A ; B)=\prod_{i=1}^{i=n} p(a i, b i) \log \frac{p(a i, b i)}{p(a i) p(b i)}
$$

The mutual information (MI) is the Kullback Leiber distance (Cover, 1991) between the joint distribution $\left[\mathrm{p}\left(\mathrm{a}_{\mathrm{i}}, \mathrm{b}_{\mathrm{i}}\right)\right]$ and the product distribution $\left[p\left(a_{i}\right) p\left(b_{i}\right)\right]$. Then $\log n$, the upper limit of $I(A ; B)$, is the maximum bound of the information channel. $\mathrm{I}(\mathrm{A} ; \mathrm{B})=\log \mathrm{n}$ can occur only when the bound of set $\mathrm{A}$ for the receiver $=\log \mathrm{n}$, and the bound of set $\mathrm{B}$ for the transmitter $=\log \mathrm{n}$, are overlapping and congruent. $\mathrm{I}(\mathrm{A} ; \mathrm{B})=\log \mathrm{n}$ only occurs when there is no noise in the communications system.

MB $\left(\log a_{n} ; \log b_{n}\right)$, the mutual bound (the lens shape enclosed in dotted lines in Figure 4), is defined as the bound of an information channel. Expanding the equation for MI above into separate joint and product entropy terms:

$$
M I=\prod_{i=1}^{i=n} p(a i, b i) \log p(a i, b i) \square \prod_{i=1}^{i=n} p(a i, b i) \log p(a i) p(b i)
$$

When $p\left(a_{i}\right)=p\left(b_{i}\right)=1 / n$, the bound of set A for the receiver $=\log \mathrm{n}$ and the bound of set $\mathrm{B}$ for the transmitter $=\log \mathrm{n}$. This is the mutual bound (MB) of the information channel. The limit of MI, which is
MB, may be found by inserting $1 / n$ for $p\left(a_{i}\right)$ and $p\left(b_{i}\right)$. Then the equation for MB is:

$$
\mathrm{MB}=-(\log n \text { to } 2 \log n)+2 \log n
$$

The product entropy term is $2 \log n$. The joint entropy term ranges from $-(\log n$ to $2 \log n)$ depending upon the Kullback Leiber distance (Cover and Thomas, 1991) which is determined by the noise in a communications system (Figure 1 ) when $a_{t}=b_{r}$ $=\mathrm{n}$.

That MB and MI both can be derived from the Kullback Leiber distance is an indication that Figure 4 presents a realistic view of the relationship of the mutual bounds to the mutual information. Using the concept of mutual bounds it is possible to examine the impact of variation of these bounds on the performance of a communications system.

\section{MODELING CONSTRAINTS}

Consider two sets that have a high probability relationship $\left(p\left(b_{i} \mid a_{i}\right)\right.$ near to 1$)$ with each other but where the number of elements in each set is different $\left(a_{t} \neq b_{r}\right)$ such as Figure 2. In the case where there is no noise in an information channel and the difference between the bounds of the two sets is the variation caused by unordered elements in sets A and B. Variation is defined as the existence of elements of set A or B that are not ordered pairs. Without variation between set $\mathrm{A}$ and set $\mathrm{B}, \mathrm{S}=\mathrm{A} \square$ $\mathrm{B}=\log \mathrm{n}$. Attempting to hold $\mathrm{a}_{\mathrm{t}}=\mathrm{b}_{\mathrm{r}}=\mathrm{n}$, thereby eliminating variation, is the practice in electronic or optical communications system design.

In an operating communications system the relationship of $a_{t}$ to $b_{r}$ to $n$, for each set of constraints is determined by the actual implementation of each set pair A and B (Figure 2). Notice when $a_{t}$ or $b_{r}>n$ the information channel is less efficient and this effect is independent of noise. As the communications system implementation approaches optimum, $\mathrm{a}_{\mathrm{t}}=\mathrm{b}_{\mathrm{r}}=\mathrm{n}$ and $p\left(\mathrm{~b}_{\mathrm{i}} \mid \mathrm{a}_{\mathrm{i}}\right)=1$ (no noise), then MB approaches $\log \mathrm{n}$ as a bound.

The term S (set of constraints), developed above, is the bound of MB. MB describes the bounds of the information channel in the presence of noise while $\mathrm{S}$ describes the bounds of an idealized information channel where noise is zero. When the noise is zero, the effect of differences in the bounds of sets $A$ and $\mathrm{B}$ on communications system performance may be examined.

Figure 4 is also useful to describe what is meant by compatibility. All sets that have any degree of 
compatibility with each other have an MB that falls within a bound $\mathrm{S} . \mathrm{MB}=\mathrm{S}=\log \mathrm{n}$ is the description of the bound on the compatibility of the two sets shown in Figure 2. A description of a communications system is often made using multiple related sets which creates a specific preexisting order (i.e., compatibility). This paper terms such a description a compatibility description as the purpose of making such a description is almost always to create or maintain compatible entities.

\section{QUANTIFYING VARIATION IN A COMMUNICATIONS SYS- TEM}

In specific designs or implementations of the transmitter and receiver (when the link characteristics are accounted for by the choice of sets $A$ and $B), a_{t}=b_{r}=n$ may not be true for each set of constraints in the system of constraints that bound a communications system. When $\mathrm{a}_{\mathrm{t}} \neq \mathrm{b}_{\mathrm{r}} \neq \mathrm{n}$ the design/implementation of the system is less than optimum. A reduction from the optimum is not necessary undesirable but it should be defined to prevent design or implementation errors. The models developed above assist in evaluating any variation from an optimum communications system design.

Multiple implementations of an actual transmitter or receiver are rarely identical. Figure 4 shows that differences in similarity directly impact compatibility. Differences in the implementations are caused by differences in the number of elements of a transmitter set $\left(a_{t}\right)$, or receiver set $\left(b_{r}\right)$ caused by some variation. Such variation (V), which is independent of noise, is caused by errors or misunderstandings in the definitions of order used (e.g., similarity or compatibility descriptions), errors in the implementations, or the implementation of different options.

The relationship between $a_{t}$ and $b_{r}$ may be used to quantify the total variation in incremental parameters. Analog parameters (non-incremental) are usually described using the concept of tolerance which defines a bi-directional variation range. In analog parameters, information variation within the specified tolerance range is ignored; cases where the information variation is beyond the specified tolerance range are considered faults in common engineering practice. For this reason this paper focuses on incremental (non-analog) parameter variation.

In the most efficient communications system design the receiver will accept all the transmitter sends and no more. This is shown as: $\log b_{r}=\log a_{t}$. The information variation is $(\mathrm{V})=\left|\log b_{\mathrm{r}}-\log \mathrm{a}_{\mathrm{t}}\right|$ for each set of constraints. The sum of the information variation of all the non-ignored non-fault sets of constraints (numbering $\mathrm{x}$ ) in a communications system is $\square \mathrm{V}_{\mathrm{i}}$ for $\mathrm{i}=1$ to $\mathrm{x}$. As $p\left(\mathrm{~b}_{\mathrm{i}} \mid \mathrm{a}_{\mathrm{i}}\right)$ goes to 1 , $M I$ and $M B$ increase. In the simplest communications system, without noise, as $\mathrm{V}$ goes to zero, $\mathrm{MB}$ goes to $\log \mathrm{n}$ as a limit, the maximum performance of the simplest communications system.

The information channel shown in Figure 2 identifies two sets (alphabets) forming the simplest communications channel. Assuming that these alphabets define only one aspect of the coding, other necessary parameters of the transmitter and receiver may include bandwidth, initialization, synchronization, training, framing, error control, compression, session layer protocol, etc. The description of these additional communications parameters entails additional sets of constraints which are each supported across an information channel such as described in Figure 2.

The difference between MB and $\log n$, not due to noise, is caused by the effects of differing implementations, defined by $\square \mathrm{V}_{\mathrm{x}}$. V terms could also include the impact of variation related to the design documentation as well as the implementations. Variation may be caused by differences in the similarity of: timer specifications, buffer sizes or revision levels (when the revisions modify the number of elements in any set in the system of constraints); and also by different options, or protocol layers, or revisions that modify the number of elements in any of these at a single end of the communications system.

When multi-protocol layer transmitters and receivers have a variation somewhere in the system of constraints, $\square \mathrm{V}_{\mathrm{x}}$ will exist as a reduction in the maximum possible MB. Given the current state of design documentation (where each set of constraints is not defined separately) the ability to compare sets of constraints in each protocol layer of a complex communications system to identify possible variation is nearly impossible. And because of the large number of combinations possible, the ability to test all possible combinations of sets of constraints is often close to impossible. Therefore, as communications systems continue to become more changeable and complex, the value of $\square V_{x}$ is 
increasing. A new mechanism, which is termed adaptability, has emerged to address this problem and decrease the effective value of $\square V_{x}$.

\section{MODEL OF AN ADAPTABLE SYSTEM}

Order in a communications system includes maintaining similarity of entities and maintaining compatibility between entities. Order may also be use to support adaptability between entities. Adaptability, as used here, is a means to negotiate the relationship between different, potentially compatible entities for the purpose of selecting the "best" pair of compatible entities required for the communications system's application.

Figure 5 shows a multi-mode communications system consisting of three independent transmitter and receiver sets and one independent etiquette. Communications is possible using any one set of the compatible transmitters and receivers. The etiquette shown in Figure 5 is used to negotiate the "best" transmitter and receiver set for a specific communications application. In this simple example higher S, which offers more possible communications states, is considered better.

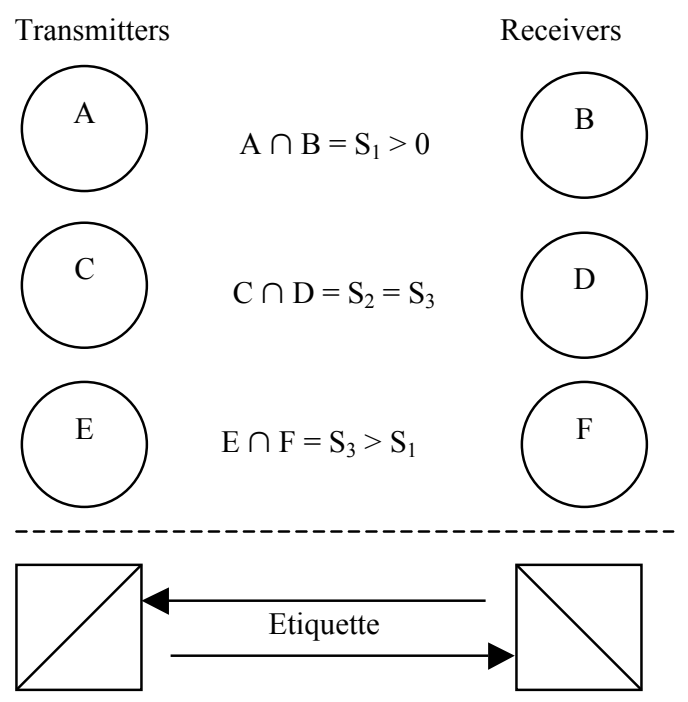

Figure 5: A multi-mode system with one etiquette.

Consider Figure 5. Transmitter set $\mathrm{A}$ and receiver set $\mathrm{B}$ are compatible. Transmitter set $\mathrm{C}$ and receiver set $\mathrm{D}$ are compatible and equal (in number of states) to transmitter set $\mathrm{E}$ and receiver set $\mathrm{F}$. Transmitter set $\mathrm{E}$ and receiver set $\mathrm{F}$ have more states than A and B. In this example none of the other possible sets are compatible. In this case it is most desirable for the transmitter and receiver selected for operation to be $\mathrm{E}$ and $\mathrm{F}$ or $\mathrm{C}$ and D. Figure 5, without the etiquette, could also be viewed as a model of a $2 \mathrm{G}$ or $3 \mathrm{G}$ tri-band cellular mobile and cellular base station. Figure 5 might also be viewed as a model of a multi-mode software defined radio (SDR).

For the purpose of creating a preexisting order, a specific description of the negotiation procedures among multiple possible information channels may be used. Such a description is an adaptability description which includes a mechanism to negotiate among multiple possible information channels to create or maintain adaptability. Such a mechanism is termed an etiquette.

\section{MINIMIZING VARIATION IN COMMUNICATIONS SYSTEMS}

Complex communications systems utilize multiple layers of compatibility standards (e.g., protocols), each of which may exhibit variation. For application to application communications to be efficient, the sum of the total communications system variation $(\square$ $\mathrm{V}_{\mathrm{x}}$ ) must be controlled, otherwise $\mathrm{MB}$ may be significantly reduced. The $\square \mathrm{V}_{\mathrm{x}}$ is very difficult to calculate in multi-protocol layer systems with timeindependent processes, and testing all possible variations is usually not practical.

Etiquettes can ensure that complex communications systems function properly at the applications layer. Etiquettes define a fully-testable independent protocol (from the data and control layer protocols) whose purpose is to negotiate among the parameters (most or all of the sets of constraints) at the transmitter and receiver to select the common sets known to fulfill the requirements necessary for a specific communications application. The purpose of an etiquette is to support adaptability.

In a communications system, multiple sets (used in multiple OSI layers) exist to define a multilayered communications interface. Changes to the sets describing the transmitter or receiver or their implementations may create elements that are not contained in the MB of a specific layer or reduce the MB of a different layer (e.g., by changing a buffer size which might reduce maximum packet length). Such changes are a cause of compatibility problems. When the changes to any compatible sets are a 
superset of the previous compatibility sets, then MB remains constant or increases. However, maintaining a superset in multiple layers of communications protocols is problematic. The ability to create a superset is made practical by requiring that an etiquette is a single tree structured protocol (which may be expanded and will always remain a superset of prior instantiations). An etiquette discovers and then negotiates between multiple transmitter and receiver implementations and their parameters at all required layers of the OSI model (X.200) to identify and select implementations that are most desirable for a required communications application. The etiquette can perform such a negotiation based on knowledge of the desired application, existing compatibility sets or even known "bugs" by using specific revisions of the sets of constraints in a desired application.

Etiquettes are already used in many communications systems e.g., ITU G3 fax T.30, telephone modems, ITU V.8, ITU digital subscriber line transceivers G.994.1, IETF Session Initiation Protocol, W3C XML; their properties have been explored in Krechmer (2000). But the value of etiquettes is not widely understood or employed. As example, the $3 \mathrm{G}$ cellular standard, IMT-2000, defines five different communications protocols. Currently the means of selecting a specific protocol stack is left to the designer. Existing multimode cellular handsets and base stations sense the strongest signal and give priority to higher generation protocols over lower (a selection mechanism). Such handsets and base stations can support protocol selection, but cannot support protocol negotiation. For a span of time, different protocol stacks will be used in different geographic areas and the negotiation that an etiquette enables is of less value. Eventually however, multi-mode cellular handsets and base stations will appear; then an etiquette becomes more important, not only to negotiate around incompatibilities that emerge as more independent implementations and revisions of the communications standards exist, but also to allow the service provider to select the protocol that optimizes system loading or optimizes geographic coverage, or to allow a user to select the protocol that offers the best economic performance. The use of adaptability mechanisms is a system architecture choice that significantly enhances the long term performance of complex communications systems.

\section{CONCLUSION}

Shannon's theory has pointed the way toward more efficient use of transmission links for over 50 years by identifying the maximum possible data rate for a given level of noise. The basic idea offered in this paper is that Shannon's theory can also point the way toward more efficient communications specifications and equipment by quantifying the effect of variation on communications systems. Utilizing mechanisms to support adaptability then offers the means to control variation in communications systems. Now that some communications designs are closing in on the maximum possible transmission link performance, it is time to address the performance gains and system improvements that can be achieved by controlling variation with etiquettes.

\section{REFERENCES}

Abramson, N. (1963). Information Theory and Coding, McGraw-Hill.

Cover, T. \& Thomas, J. (1991). Elements of Information Theory, John Wiley \& Sons, Inc.

Kant, I. (1800). Logic, first published 1800 (General Doctrine of Elements, para. 6, Logical Acts of Comparison, Reflection and Abstraction).

Krechmer, K. (2000). Fundamental Nature of Standards: Technical Perspective, IEEE Communications Magazine, Vol. 38 No. 6, p. 70, June. Retreived from http://www.csrstds.com.

Russell, B. (no date). Introduction to Mathematical Philosophy, page 14, Simon and Schuster.

Shannon, C. E. \& Weaver, W. (1963). The Mathematical Theory of Communications, page 58, Theorem 8, University of Illinois Press, Urbana and Chicago IL, USA. 